\title{
TREATMENT OF CONVERGENT SQUINT ASSOCIATED
} WITH HYPERMETROPIA*

BY

\author{
Grace VAughton aND MaUREen STEWART \\ From the Orthoptic Department, Nottingham and Midland Eye Infirmary.
}

For many years we have made, under the surgeons with whom we have worked, a study of the most beneficial glasses that can be ordered in conjunction with orthoptic treatment, because we have felt that the success of orthoptic treatment depends upon the glasses ordered, and we are convinced that glasses are the basis of orthoptic treatment.

In cases of convergent squint associated with hypermetropia, experience has shown us that, when the relationship between convergence and accommodation breaks down, the patient makes an excessive convergence effort to obtain clear vision uniocularly and, if diplopia has been present, to separate the images far enough for them not to be confusing. The suppression of the second image is thus facilitated. The breakdown between accommodation and convergence is primarily caused by accommodation exerted disproportionately to convergence in order to overcome the hypermetropia, and this breakdown is probably due to a deficiency in the power of relative accommodation, namely, the ability to accommodate in excess of convergence.

Most children are hypermetropic; it is not known exactly why this refractive state should induce squint in a minority of cases, but it may well be that deficiency in the power of relative accommodation is the decisive factor in producing deviation.

Ability to maintain clear binocular single vision at any distance is the hallmark of a normal pair of eyes, and as this function is mainly accommodational, the defect in accommodation must first engage our attention in cases of squint. We are convinced that, by readjusting accommodation, we are laying the foundation of a cure. Otherwise there is bound to be a weakness in the relationship between the two eyes. The ciliary muscle is therefore like a sick person who has to be nursed back to health and normal activity, gradually and without undue strain.

In order to rest and relax the ciliary muscle it is necessary to correct manifest and latent hypermetropia. Only when the need for accommodation is thus reduced, can the excessive convergence effort undergo relaxation.

To ascertain the necessary correction to do this, we have found that patients should have their accommodation completely paralysed by a cycloplegic (such as atropine), so that retinoscopy may

\footnotetext{
- Received for publication December 14, 1949.
} 
reveal all the latent hypermetropia. Suppose retinoscopy is carried out at one metre distance, then full correction implies that the child should be ordered the shadow-turning lens less one dioptre, and without further subtraction for the cycloplegic used. Any astigmatism present should also be fully corrected. Many people claim that no child will tolerate such a full correction, but in our experience we have not found this to be so. The child should be corrected as soon after the onset of squint as possible. When a child has been under-corrected for a few years, it may be necessary, in a few cases, to maintain atropinization for about a week when the glasses are first worn. He will then wear them without any difficulty.

We realize that this correction is not generally used in all orthoptic clinics, and our object in writing this paper is to show why we think it is an integral part of the treatment. We believe that a full correction is necessary in order to overcome the excessive convergence effort. Many children appear to be straight in glasses, but converge one eye on being asked to fix a distant or near object. Questioning frequently reveals the fact that, when their eyes are straight, these children tolerate slightly misty vision for distance or near, or for both, but can overcome this by accommodating and consequently converging. By adding extra plus-spheres we have been able to clear the child's vision while the eyes are kept straight, and this in turn has prevented the child from over-converging for distance or near work. The lens that does this is the full correction by previous definition-that is, the lens that corrects the total hypermetropia. One of the most difficult cases with which an orthoptist has to deal is that of the child who is straight and orthophoric for distance, but over-converges one eye for near work. Orthoptists will find that the above 'method of correction will eliminate the majority of these cases.

Quoting from Lyle and Jackson (1949), it seems that what we would term a "full correction" they would term an "overcorrection ". They say " . . . it is important to test the binocular visual acuity. This of course only appertains in those cases in which the visual axes are parallel for distant vision. It may be found that a better binocular visual acuity is obtained, in cases of hypermetropia especially, by the further addition of plus-spheres in front of each eye, in which case such additional plus spherical correction should be prescribed". We are confident that this correction should be prescribed in all cases of convergent squint.

We have found in our work that a child whose accommodation has not been entirely relaxed by the glasses tends to develop a small convergent angle of squint. This remains, and the squint becomes of the tonic type with an accommodative element. By 
tonic we mean that natural adaptation of the muscles which has resulted from the active contraction of one muscle and the reciprocal relaxation of its antagonist. This excessive convergence effort results in suppression, which is greatly reduced immediately the need for the convergent effort is removed by a full correction. This is a tremendous factor towards gaining a satisfactory cure, as suppression plays such a large part in orthoptic treatment.

Apart from reducing the suppression, it will be found that this correction will straighten a large percentage of squints, and it should also be prescribed in the unilateral and alternating types of squints where operation is necessary. It is equally important to relax excessive convergence effort as suppression is reduced and muscle-tone is improved in these cases, and there is more chance of developing binocular single vision after the operation has been performed.

We are convinced that removing this excessive convergence effort is the first step in curing a squint. This seems to be substantiated by the fact that amongst the unilateral and alternating types of squints there are pre-operative cases who have barely simultaneous perception and do not respond to treatment. When first tested on the synoptophore ten days or so after operation, a number of these cases show definite signs of binocular vision which is rapidly improved with treatment, so that they become perfect cures. We know that if the eyes are put straight by operation there is a chance of binocular single vision developing, but we feel that this is not only because the eyes are straight but also because the excessive convergence effort has been relaxed. This has been further proved by the fact that, though the eyes may be put straight by operation, yet, because a small convergent effort is still present owing to the glasses being too weak, there is a tendency for the eyes to diverge or converge rather than to settle down around zero and develop fusion. Such cases show intense central suppression. This may be illustrated by the following

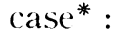

Case 1. T. W. (aged 101 years).

Defect.-Original R.C.S. Straight post-operatively.

Age of onset. -2 years.

History.-Ciradual onset, attended clinic.

Partly dilated, R. and L. -+4.5

Ordered R. and L. +2.0 D. sph.

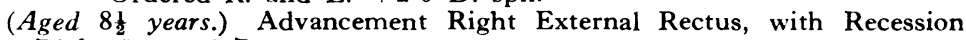
Right Internal Rectus.

$$
\begin{aligned}
& \begin{array}{llll}
\text { (Aged } 9 \frac{1}{2} \text { years.) } & \text { Refracted R. }-6.25 & +6.25 \\
-+6.25 & \text { L. }-6.75
\end{array} \\
& \text { Ordered R. +4.5 D. sph. L. } \frac{+4.5 \text { D. sph. }}{+0.5 \text { D. cyl. } 90^{\circ}}
\end{aligned}
$$

\footnotetext{
* Retinoscopies given in all examples are the shadow-turning lenses.
} 
1 st Test. (Aged $10 \frac{1}{2}$ years.) R.V. $\bar{c}$ gls. $=6 / 12$. L.V. $\bar{c}$ gls. $=6 / 6$.

Synoptophore. -Angle $\overline{\mathrm{c}}$ gls. $=0^{\circ} /-6^{\circ}$. Angle $\overline{\mathrm{s}}$ gls. $=+12^{\circ}$.

S.M.P.-Very unsteady, showing marked right suppress ion. Right image always moving.

Fusion.-No.

Off the instrument the child appeared straight during conversation, but he diverged the right eye when looking in the distance, and had no power of convergence. His pupils were enlarged and showed no sign of contraction except to a light.

In spite of working hard he showed no response to treatment. In order to prevent the divergence and to encourage the power of convergence his glasses were reduced, but in these he then converged during conversation. It was impossible to find a lens in which he did not either diverge or converge, and the treatment did not remove the intense central suppression or steady the right image.

It seems from this and similar cases that the accommodational defect must be corrected both by glasses and treatment before operation. Otherwise great difficulty is experienced by the patient in adjusting his eyes to obtain binocular single vision.

Chavasse (1937) says: "The probability that the normal developmental changes in the refraction of the eyes are influenced by adaptive use or disuse should be borne in mind in prescribing glasses for any growing child. The factors which secure that infantile ametropia normally issues in adult emmetropia are not yet defined. But the possibility that the wearing in childhood of a 'correction' for a normal degree of ametropia may influence adversely the attainment of adult emmetropia is ever present.

" The strength of the glass ordered should therefore be the weakest which keeps the squint in the occasional category. As soon as, in glasses, the squint is never present, the strength of the glasses must be further reduced. Otherwise the development of the harmonic accommodation reflex is obstructed, so that, when growth is complete, the glasses cannot be removed without precipitating the squint".

We do not feel that we can agree with this statement, because we have worked for many years with cases that have been " fully corrected"' and we have never found any ill-effects developing then or in later years : on the contrary, these cases have responded better to orthoptic treatment. The correct relationship between accommodation and convergence has been more quickly established, and when the child has been " discharged as cured" following the treatment, his glasses are then, and only then, periodically reduced.

In children who are " under-corrected" as we would term it, we have found marked disturbance of accommodation. It would seem that these children suffer from fatigue of accommodation resulting from the continual necessity to see clearly, and the power 
of accommodation becomes faulty in consequence. This fatigue manifests itself in (1) enlarged pupils which contract to a light and do not do so to accommodation, and (2) lack of proper convergence. The child is able to read at a normal distance, but he is conscious of seeing better at a slightly greater distance. These cases rarely become a satisfactory cure because their fusion is faulty, and their adduction almost nil, and there is little real relationship between the two eyes.

To illustrate the importance of the functioning of accommodation we quote the following example:

Case 2. S. B. (aged $7 \frac{3}{4}$ years).

Defect.-Original R.C.S. Straight post-operatively.

Age of onset. $-4 \frac{1}{2}$ years.

History.-C ( iradual onset, attended clinic.

$$
+5.25+4.75
$$

Refracted R. - +525 L. --+475

Ordered R. +2.5 D. sph. L. $+2 \cdot 0$ D. sph.

(Aged 73 years.) Advancement Right External Rectus with Recession Right Internal Rectus. Immediately after operation referred for treatment.

1st Test.-While in-patient.

Synoptophore.-Angle $\bar{c}$ gls. $=+5^{\circ}$. Rt. Lt. $2 \mathrm{~A}$.

S.M.P.-Spasmodic, but never held, pictures " shoot across" most of the time.

Fusion.-No.

Off the instrument this child was quite straight with no cover test, but he had enlarged pupils and no power of convergence. We treated him on the synoptophore in the usual manner, but without response. We were unable to steady the S.M.P. or to develop fusion. We then started an attack on the pupils by working with plus- and minus-lenses on and off the synoptophore, making him look into the distance and converge to a near finger, and indeed anything that would alter the size of the pupils. At first we met with no response. The child had no convergence to a finger, and in order to "clear", even a - 1.0 D. sph. in front of each eye, he pushed one eye "in" instead of accommodating. During the second treatment of this kind we noticed improvement. There was a slight contraction of the pupils and a better attempt at convergence. The third treatment showed further improvement, and when he was tested on the synoptophor: he showed definite signs of fusion, and was altogether steadier. This has improved, and the child is now responding well to treatment.

These cases with fatigue of accommodation are difficult to improve, but we have found the above method very satisfactory. We are able thus to cure some of these cases, which would formerly have been discharged as " cosmetic" cures.

The following practical points illustrate our argument in favour of full correction :

(1) The need for excessive accommodation is removed, and the facility with which the child over-converges is curtailed.

(2) Suppression is greatly reduced.

(3) The angle of squint is removed unless there is any tonic element, and the case can be cured by treatment alone.

(4) Full correction greatly minimizes the chance of an abnormal retinal correspondence developing. 
Orthoptists know that there are certain conditions that are unresponsive to orthoptic treatment, but we believe that a full correction from an early age is particularly beneficial in the following types :

(1) Variable angles of squint.

(2) Cases of over-convergence when fixing a distant object.

(3) Cases of over-convergence when fixing a near object.

(4) Small convergent angles with no binocular vision who attempt a false associated fixation at zero.

(5) Cases of intense central suppression which do not yield to treatment. These, at the best, will only be a semi-orthoptic cure if any fusion can be obtained.

(6) Cases having simultaneous perception but unable to develop fusion owing to the continual slight slipping "off" of one eye. Inexperienced orthoptists might work on and develop a slightly false fixation in these cases.

(7) Enlarged pupils which do not contract during an effort to accommodate or converge, associated with absence of normal adduction and convergence.

From the age of onset our aim is, if possible, to maintain binocular single vision with the aid of glasses until the child is old enough for treatment. We find that a full correction is the best way of doing this, because there is more chance of the eyes being kept straight all the time, and less chance of suppression developing.

It has been interesting to note that children on regular treatment who have been, as we would term it, under-corrected, show a constant or occasional angle of squint with marked suppression. Sometimes these children have barely simultaneous perception, but, as extra plus-lenses are added on the synoptophore, this suppression is immediately reduced and simultaneous macular perception obtained. If the extra correction can be given soon after the onset of squint, the small convergent angle or variable angle will be overcome. If the small angle of convergence is allowed to remain, and if it becomes consistent, abnormal retinal correspondence with marked suppression around the true angle is likely to ensue. We believe that these are the cases of abnormal retinal correspondence which orthoptists claim to overcome in a few treatments. In such patients operation may immediately secure binocular single vision. In reality these are cases of undercorrected accommodative squint with a desire for fusion. Most of them, if a full correction had been prescribed, would never have developed a small convergent angle at all, but would have maintained weak binocular single vision which could have been perfected by treatment at a later stage.

Some of our observed cases of squint with a small static convergent angle in glasses have become quite straight when the glasses were removed, and they even tolerated misty vision. As soon as the glasses were put on again, the static convergent angle reappeared, because the child had found that, by maintaining a 
small amount of convergence, he could see quite clearly in his glasses. By this effort of convergence he was overcoming the residual uncorrected hypermetropia. By giving him this extra correction we remove the static angle, and he can see clearly in his glasses with his eyes straight. We do not want this type of squint to be confused with the accommodative type which converges on fixing a distant or near object.

The following cases illustrate, from the orthoptic point of view, the advantages of a full correction.

Case 3. M. S. (aged $6 \frac{1}{2}$ years).

Defect.-R.C.S. variable, converging more on fixing.

Age of onset. -5 years.

History:-Gradual onset, attended clinic.

Refracted. R. and L. -+5.5

Ordered R. and L. $+3 \cdot 0$ D. sph. Referred for pre-operative treatment.

1 st Test (aged $6 \frac{1}{2}$ years).-R.V. $\bar{c}$ gls. $=6 / 12$ pt. L.V. $\bar{c}$ gls. $=6 / 12$.

Synoptophore. - Angle $\bar{c}$ gls. $=+20^{\circ}$ variable. Angle $\overline{\mathrm{s}}$ gls. $=+40^{\circ}$ variable. S.M.P. - Yes, variable, with marked right suppression.

Fusion.-Seemed to be present, but was variable and unsteady, and showed marked right suppression.

Quoting from report, "On the instrument the angle is much reduced by adding a $+2.0 \mathrm{D}$. sph. each eye, and child can join fusion pictures at $+5^{\circ}$, holding both control marks, whereas with her own glasses she joins the pictures at $+15^{\circ} / 18^{\circ}$ and loses the right control, showing how much the suppression is increased by accommodation".

This child was then ordered R. and L. +4.5 D. sph., in which she was quite straight with all the grades of binocular vision, and she was discharged as an orthoptic cure without operation after 16 treatments.

Case 4. M. C. (aged 8 years).

Defect.--L.C.S. can alternate.

Age of onset. -10 months.

History.-Measles. Attended clinic immediately.

$$
\begin{array}{llll}
\text { (Aged 2 } 2 \frac{1}{2} \text { years.) } & \text { Refracted R. }-+7.0 & +8.5 \\
-7.0 & \text { L. }- & -+8.5
\end{array}
$$

Ordered R. +4.5 D. sph. L. +6.0 D. sph.

1 st Test (aged 8 years). - R.V. $\bar{c}+4 \cdot 5$ D. sph. $=6 / 5$. L.V. $\bar{c}+6$ D. $s p h=6 / 5$.

Synoptophore.-Angle $\bar{c}$ gls. $=+20^{\circ}$, very variable. ' No simultaneous perception.

S.M.P.-No.

Fusion-No.

After three treatments child only obtained spasmodic simultaneous perception. She was refracted again and was ordered a full correction, namely R. $+5 \cdot 5 \mathrm{D}$. sph. L. +7.0 D. sph. With this correction the suppression was greatly reduced and she had comparatively steady S.M.P. at about $+5^{\circ}$. After five treatments she could join large fusion pictures, steadily holding both controls all the time, but with one dioptre less in each eye, the fusion hold became weak, and the right control kept coming and going. She gradually became quite straight, and capable of fusion, ductions and stereoscopic vision, but she is still under observation, as at times she has misty vision for near work when maintaining binocular single vision, owing to fatigue of accommodation; but this is improving. The pupils, which were very enlarged, are becoming normal. 
Case 5. J. H. (aged 7 years).

Defect-L.C.S. Appearance $+15^{\circ} \overline{\bar{c}}$ glasses.

Age of onset. -3 years." $\quad+25^{\circ} \bar{s}$ glasses.

History-Occasional squint, gradual onset, attended clinic immediately. Refracted R. $-\mid++5 \cdot 0 . \quad$ L. $\quad-\mid++5.5 . \quad$ No glasses ordered.

(Aged 4 $\frac{1}{2}$ years.) Constant L.C.S.

Refracted R. and L. $-\mid-+5.5$

Ordered R. and L. +2.5 D. sph.

(Aged 7 years.)

Refracted R. and L. -+5.5

Ordered R. and L. +3.0 D. sph.

1 st Test (aged 7 years).-R.V. $\bar{c}$ gls. $=6 / 6$. L.V. $\bar{c}$ gls. $=6 / 9$ pt.

Synoptophore.-Angle $\bar{c}$ gls. $=+18^{\circ}$. Angle $\overline{\mathrm{s}}$ gls. $=+25^{\circ}$.

S.M.P.-Yes, with marked left suppression.

Fusion.-No.

. Little response to treatment was shown. Her correction was increased to a full correction, i.e., $R . \bar{c}+4.5$ D. sph. $=6 / 5$ pt., L. $\bar{c}+4.5$ D. sph. $=6 / 6$ pt., but it was too late after onset to be effective, and the angle of squint remained. Suppression had become too intense as a result of the excessive convergence effort to respond to treatment.

Although she has the history of a typical accommodative squint, she had to have an operation to remove the angle, and after further treatment-34 attendances in all-she was discharged as cosmetically straight.

It seems that if this child had been fully corrected from the age of onset she would probably have been a typical accommodative squint curable by treatment alone. She also had enlarged pupils.

We should like to comment here on what have been termed emmetropic accommodative squints, because we have never found a case that would fit into this category. From the practical point of view of trying to cure the squint, we have always found that, by temporarily correcting the latent hypermetropia however small, glasses have eliminated over-convergence, and the case has become a typical accommodative, able to discontinue glasses within a reasonable time after a cure has been effected.

Case 6. C. F. (aged $8 \frac{1}{2}$ years).

Defect.-Occasional R.C.S.

Age of onset. $-4 \frac{1}{2}$ years.

History.-No glasses worn; received orthoptic treatment elsewhere. Referred to us for continuance of treatment. Report stated child sometimes saw mistily when eyes were straight.

1 st Test (aged $8 \frac{1}{2}$ years).-R.E. $=6 / 9$ pt. L.E. $=6 / 6$ pt.

Synoptophore.-Angle $=+5^{\circ} / 8^{\circ}$.

S.M.P.-Yes, with right suppression.

Fusion.-Yes. Adduction weak, shows right suppression.

Stereoscopic vision.-Weak.

This child was refracted and was found to be R. and L. -+2.5 ordered R. and L.+1.0 D. sph. With these the squint was less apparent and she was perfectly comfortable in them. 
Synoptophore (after 8 treatments).-Angle $\bar{c}$ gls. $=+4^{\circ} / 5^{\circ}$. Angle $\bar{c}$ out gls. $=+4^{\circ} / 5^{\circ}$. No suppression apparent.

S.M.P.-Yes.

Fusion.-Yes. Adduction. $-20^{\circ}$. Abduction.- $3^{\circ}$.

Stereoscopic vision.--Yes.

Physiological diplopia.--Yes.

She has maintained improvement, but when she started treatment she had a marked fatigue of accommodation. When this has fully recovered she will be able to discontinue glasses.

In our method of giving treatment closely allied with the treatment of the accommodational element, we believe that it is equally important to train the adduction immediately fusion develops. Accommodation and convergence are associated functions; it is therefore reasonable to suppose that one helps the other, and a normal relationship is thus more quickly established. We have found that by concentrating on the adduction training and ignoring abduction, fusion has become " strong" more rapidly, suppression is eliminated, and stereoscopic vision developed. Unless carefully watched, abduction training can pull eyes out on to a slightly false fixation, but this danger is avoided if the fusion has first been strengthened by adduction exercises.

It appears that this method of treatment is not universal. Many people fear that it might increase the angle of convergence, but we do not find this to be so. We use it in all cases of squint with fusion, both pre-operative and post-operative. Once good adduction on the instrument and normal convergence off the instrument have been obtained, there is little fear of a relapse.

Some years ago we studied the correction of refractive errors in cases of squint, particularly in young children soon after onset. We recorded a consecutive series of young children attending the Out-Patient Department for the first time shortly after the squint was noticed. All these children were refracted under atropine, given a full correction, and told to report after wearing the new glasses for a month. Of 124 children who returned, it was interesting to find that 89 were straight in their glasses, and the remaining 35 still showed some deviation. The fact that 72 per cent. were straightened by glasses proved our methods of treatment were worthy of further investigation. Our subsequent experience in treating some of the above cases, when they were old enough, and others similarly corrected, has left us in no doubt that successful orthoptic treatment of concomitant squint depends largely upon the full refractive correction being ordered as soon as the deviation becomes manifest.

\section{REFERENCES}

Chavasse, F. B. (1937). "Worth's Squint". 3rd edition. Baillière, London. p 495 .

LYI.F, T K., and JACKSON, S. (1949). "Practical Orthoptics in the Treatment of Squint". H. K. Lewis, London. p. 82. 